\title{
Clinical Evaluation of the Use of Tibial Bone Grafting in Dentoalveolar Reconstructive Surgery
}

\author{
Fethi Atil $^{\mathrm{a}}$ Ismail Doruk Kocyigit ${ }^{\mathrm{a}}$ Berkay Tolga Suer ${ }^{\mathrm{b}}$ Yunus Emre Alp ${ }^{\mathrm{a}}$ \\ Mürüde Yazan ${ }^{a}$ Umut Tekin ${ }^{a}$ Hakan H. Tuz ${ }^{c}$ \\ a Department of Oral and Maxillofacial Surgery, Faculty of Dentistry, University of Kirikkale, Kirikkale, ${ }^{\mathrm{b}}$ Department \\ of Oral and Maxillofacial Surgery, Gulhane Military Medical Academy Haydarpasa Teaching Hospital, Istanbul, and \\ 'Department of Oral and Maxillofacial Surgery, Faculty of Dentistry, University of Hacettepe, Ankara, Turkey
}

\section{Key Words}

Autogenous bone graft · Tibial bone · Dentoalveolar surgery

\begin{abstract}
Objective: The aim of this study was to evaluate the efficacy of tibial autogenous bone grafting in the treatment of patients with alveolar bone defects. Materials and Methods: The study subjects consisted of 12 patients ( 10 male, 2 female, age: 19-51 years) who underwent reconstructive autogenous bone-grafting procedures. The medial approach to the tibial bone was used to harvest autogenous cancellous bone grafts in all the patients. Clinical parameters (complications at the donor and recipient sites, resorption and volume of the grafts) were evaluated retrospectively. $\boldsymbol{R e}$ sults: The mean age of the patients was $36.25 \pm 0.9$ years. Of the 12 patients, $5(41.7 \%)$ received bone grafts for sinus augmentation, $3(25 \%)$ for cyst cavity reconstruction and 4 (33.3\%) for alveolar cleft reconstruction procedures. The average follow-up period was 28.4 months (range: 21-40 months). An average of $5.2 \mathrm{~cm}^{3}$ of cancellous bone was harvested for grafting procedures. All the grafting procedures were successful, and there were no surgical complications
\end{abstract}

during the harvesting protocol. In all cases, pain and gait disturbance lasted less than 2 weeks. Conclusion: The results of this study suggest that the use of tibial autogenous bone graft harvested using a medial approach was a safe, simple and effective method for grafting various alveolar bone defects where high amounts of cancellous bone grafts were needed with low morbidity.

(c) 2015 S. Karger AG, Basel

\section{Introduction}

The use of autogenous bone grafts is relatively common in the reconstruction of bone defects of the maxillofacial region. The amount of graft required is effectively determined by the size and shape of the defect [1]. Typical intraoral donor sites include the mandibular ramus, corpus, symphysis, coronoid process and maxillary tuber region, whereas extraoral harvesting sites include the iliac crest, proximal tibia, costa and calvarium [2]. Each of these regions poses advantages and disadvantages related to bone characteristics and amount of bone required for harvesting. In comparison to extraoral regions, intraoral donor sites involve relatively lower morbidity rates due to

\begin{tabular}{ll}
\hline KARGER & $\begin{array}{l}\text { ( ) 2015 S. Karger AG, Basel } \\
1011-7571 / 15 / 0251-0072 \$ 39.50 / 0 \quad \text { Karger }\end{array}$ \\
$\begin{array}{l}\text { E-Mail karger@karger.com } \\
\text { www.karger.com/mpp }\end{array}$ & $\begin{array}{l}\text { Thisis an Open Access article licensed under the terms of the } \\
\text { Creative Commons Attribution-NonCommercial 3.0 Un- } \\
\text { ported license (CC BY-NC) (www.karger.com/OA-license), } \\
\text { applicable to the online version of the article only. Distribu- } \\
\text { tion permitted for non-commercial purposes only. }\end{array}$
\end{tabular}

Yunus Emre Alp

Department of Oral and Maxillofacial Surgery

Faculty of Dentistry, Kirikkale University

TR-71100 Kirikkale (Turkey)

E-Mail yunusemrealp@yahoo.com 
the simplicity of the surgical procedure, which involves a single area for both donor and recipient sites. However, intraoral sites may not provide sufficient graft material to repair larger defects. Conversely, extraoral autogenous bone graft sites can provide grafts of sufficient size for the repair of maxillofacial bone defects, but involve more extensive surgery, including the creation of a second surgical site and a lengthening of the duration of the surgical procedures, as well as higher morbidity rates [3].

The use of the tibia as a donor site was first described by Drachter in 1914 as reported by Winsenburg [4]. Compared to other extraoral bone graft donor sites, the tibia has lower morbidity and complication rates and requires a relatively simpler surgical technique $[3,5]$. Currently, two different techniques are used to harvest grafts from the proximal tibia, namely the medial and lateral approaches [6].

The aim of the present study was to report on the clinical advantages, disadvantages and complications of tibial bone graft harvesting via the medial approach as well as the subsequent use of the acquired grafts in the augmentation procedures of dentoalveolar defects at our institution.

\section{Subjects and Methods}

This retrospective study comprised 12 patients who underwent reconstructive surgery to repair alveolar bone defects with autogenous grafts harvested from tibial bone using a medial approach. The tibial bone was used as a donor site for maxillary sinus floor augmentations, reconstruction of alveolar clefts and repairs of alveolar defects caused by enucleation of an odontogenic cyst prior to dental implant placement (table 1). Patients were informed about surgery, postoperative recovery and possible complications. The study was conducted in line with the Helsinki Declaration on medical protocol and ethics, and all participants (and, in the case of minor patients, parents) signed an informed consent form. All patients were systemically healthy (ASA I) and had no physical deformities. Indications, donor and recipient site morbidity rates, surgical and postsurgical complications and volumes of graft material obtained were recorded for all patients. Radiological data were obtained during the course of follow-up (range, 21-40 months), beginning 2 months after surgery in order to assist in evaluating the success of the grafting procedures.

\section{Surgical Procedure}

The dental chair was adjusted to the supine position to facilitate access to the donor site, and a rolled-up sterile support was placed at the popliteal fossa in order to maintain the knee joint in flexion. The surgical area was prepped and draped in a routine surgical manner, and then local anesthesia (articaine 4\%:adrenaline $1: 100,000)$ was administered through 2 injections; the first into the medial condyle region, and the second into the periosteum. After identifying the major anatomical regions, a sterilized marker was

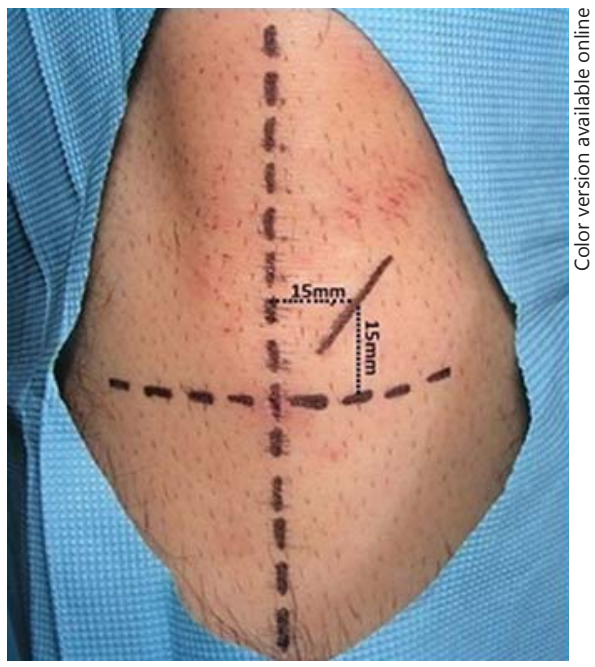

Fig. 1. Marking the incision line at the donor site.

Table 1. Distribution of the use of cancellous bone graft harvested from the tibia for this study

\begin{tabular}{lc}
\hline Procedure & Patients \\
\hline $\begin{array}{l}\text { Repair of alveolar fissure defects } \\
\text { Sinus floor elevation }\end{array}$ & $4(33.3 \%)$ \\
$\begin{array}{l}\text { Repair of defects caused by enucleation of } \\
\quad \begin{array}{l}\text { an odontogenous cyst prior to a dental } \\
\text { implantation }\end{array}\end{array}$ & $5(41.7 \%)$ \\
\hline Total & $3(25 \%)$ \\
\hline
\end{tabular}

used to draw a 3-cm-long oblique incision line starting $15 \mathrm{~mm}$ above the horizontal line passing through the tibial tubercle and $15 \mathrm{~mm}$ medial to the vertical axis (fig. 1).

Following skin incision, dermal and subdermal tissues were dissected to access the supraperiosteal plane. A trapdoor technique was used in which 2 vertical cuts of $1-2 \mathrm{~cm}$ in length were made over the crest using oscillating saws and were joined together with a horizontal cut in the medial surface. Angled blade osteotomes were used to pry out the resulting bony window in a lateral direction to create a controlled fracture and obtain a maneuverable window of cortical bone (fig. 2a) that was opened laterally to expose the cancellous bone cavity. Bone curettes were used to harvest enough cancellous bone to reconstruct the recipient site (fig. 2b). After a sufficient amount of graft material had been obtained and placed in sterilized tubes, the cavity was washed with a jet of normal saline solution, all remaining bone fragments were aspirated, and sharp edges were rounded using milling cutters and bone files. Intraoperative information with regard to the amount of collected bone was recorded.

After achieving hemostasis, the periosteal tissue surrounding the bony window was replaced in its former position and stabilized with interrupted 3-0 resorbable sutures. Subcutaneous tissue was 
Fig. 2. Bone curettes were used to obtain cancellous bone graft (a), which is transferred to the recipient site in sterilized surgical basins (b).

Fig. 3. Radiographic examination 3 months later after the augmentation procedure.

Fig. 4. Radiographic examination after the implantation procedure.
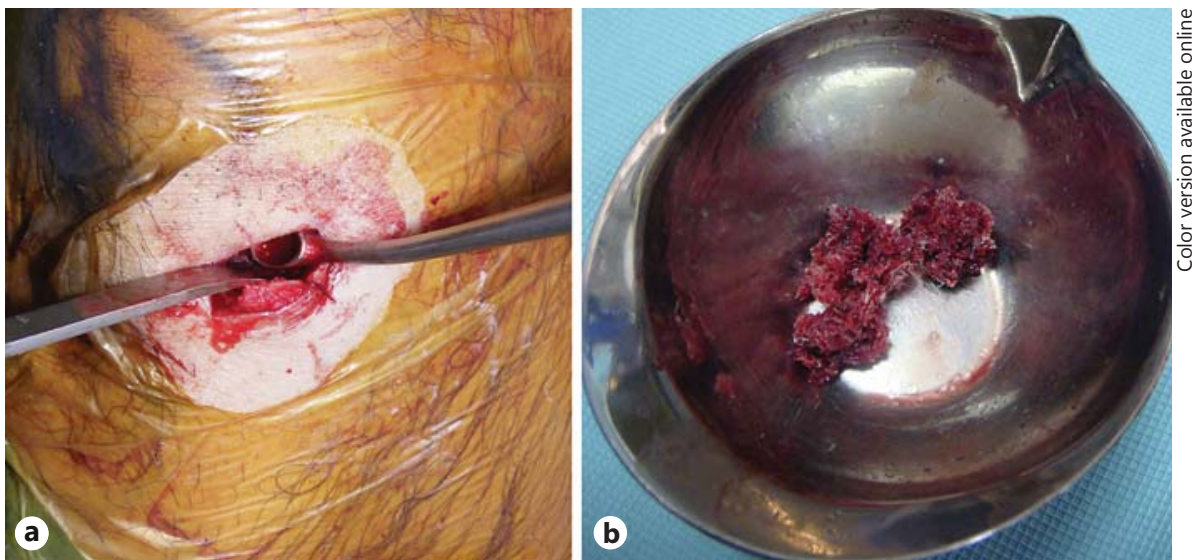

4
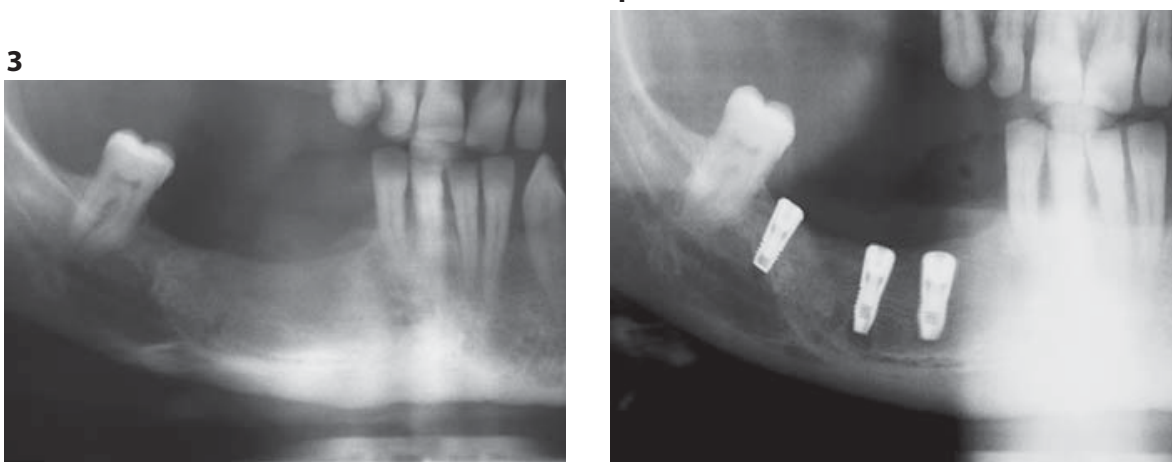

reapproximated with 3-0 resorbable sutures, and the skin was closed with 4-0 prolene sutures.

The harvested tibial bone graft was used for sinus bone-grafting procedures in 5 cases, for alveolar cleft reconstruction in 4 cases and for reconstruction of cyst enucleation cavities prior to implant placement in 3 cases. After all recipient sites had been grafted with harvested tibial cancellous bone, a resorbable collagen membrane was used to cover the augmented areas, and primary closure was obtained in a tension-free manner. Patients were discharged on the same day following a 3-hour postsurgical observation period.

Over a 7-day recovery period, patients were administered oral antibiotics and nonsteroid anti-inflammatory drugs as well as a $2 \%$ chlorhexidine gluconate mouthwash for intraoral wound care. Medical dressings of leg wounds were changed at 2-day intervals. Patients were also advised to apply cold compresses to both surgical sites during the first $24 \mathrm{~h}$ after surgery and to refrain from walking long distances and engaging in strenuous physical activities such as climbing stairs or sports activity for at least 3 months. Recipient sites were examined clinically and radiographically 3 months after surgery (fig. 3). All patients were followed weekly and examined for any gait disturbances or wound complications.

In the cases of alveolar defects caused by cysts, dental implant surgery was performed 3 months after the enucleation and the reconstruction (fig. 4), whereas in the cases of sinus floor augmentation and alveolar cleft repair, dental implant surgery was performed after a 4 -month waiting period. The postoperative followup ranged from 21 to 40 months.

\section{Results}

In all 12 cases, patients were discharged on the day of surgery. There were no complications either intraoperatively or immediately postoperatively that called for extension of hospitalization. The mean follow-up period was $28.4 \pm 7.3$ months. Minimal scar formation of only minor aesthetic concern was observed 1 year following tibial bone harvest procedures at the donor sites (fig. 5). The mean volume of harvested compressed autogenous bone volume obtained per site was $5.2 \pm 1.6 \mathrm{~cm}^{3}$ (range, $3-8$; table 2). All in all, 12 defect areas in 12 patients were treated with tibial autogenous cancellous bone grafts. Of the 12 reconstruction cases, 5 were treated for sinus floor augmentation (fig. 6), 4 for alveolar clefts (in cleft lip and palate patients) and 3 for postenucleation defects in odontogenic cyst patients (fig. 7).

Two minor complications were observed at recipient sites $(16.6 \%)$, both of which involved an infection that developed at a site where a graft was planted to facilitate the repair of an alveolar defect caused by cyst enucleation. In each case, partial loss of the bone graft was incurred; the partially infected graft material was removed, and 
5

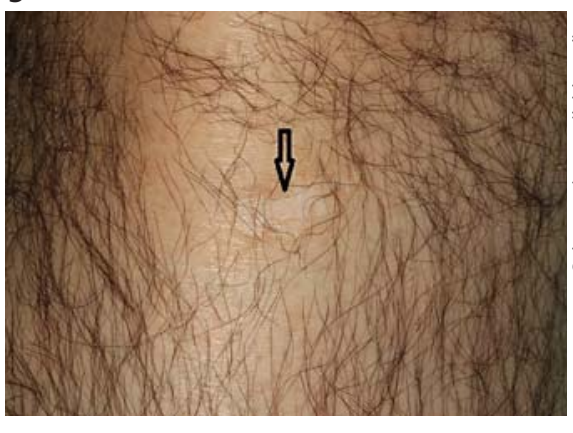

6

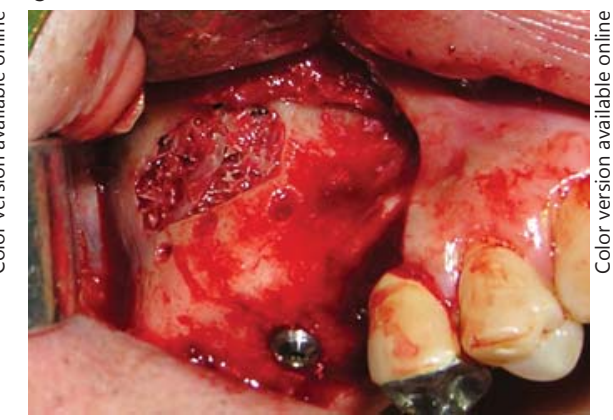

Fig. 5. Scar formation at the donor region 1 year after the operation.

Fig. 6. Sinus augmentation with cancellous bone graft harvested from the tibia.

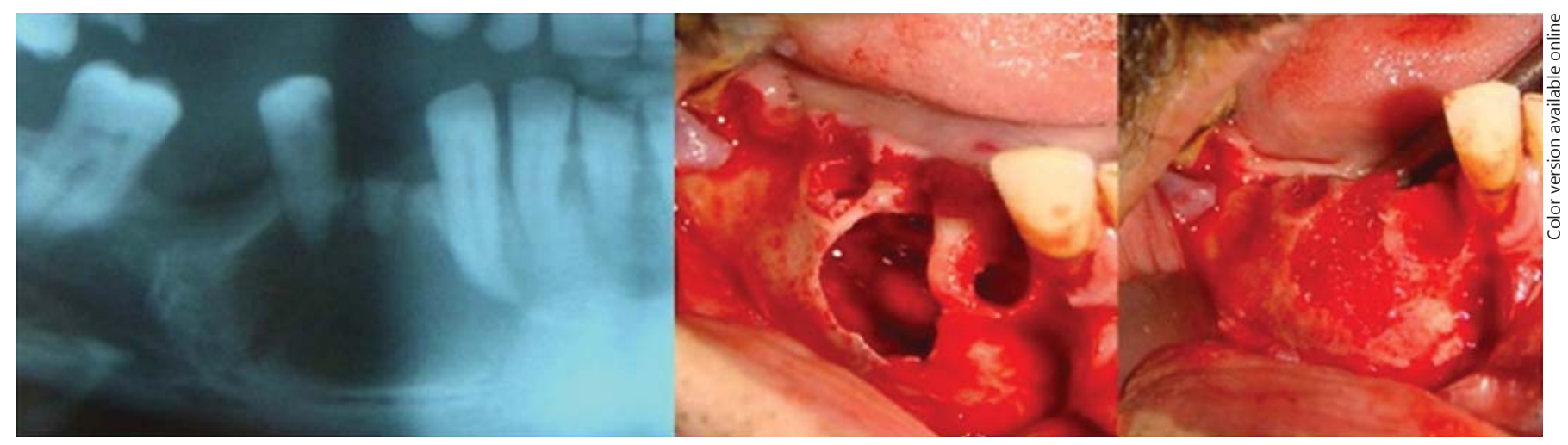

Fig. 7. Cancellous tibial grafts were used to repair a defect caused by enucleation of an odontogenic cyst.

Table 2. Patient demographic and treatment information

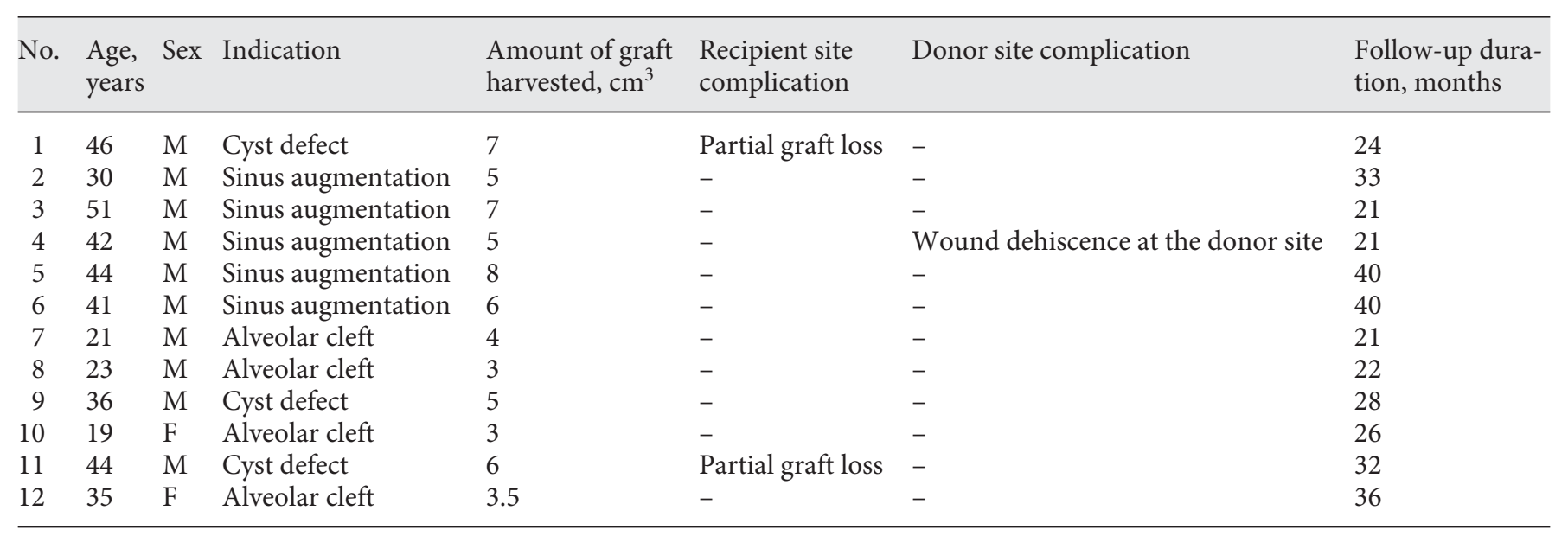

supplemental allogenic graft material was placed at the site in a second surgical operation. The postoperative follow-up showed that both cases healed without any further problems, and the desired amounts of bone were obtained for implant placement.

Tibial Bone Grafting in Dental Surgery
Only 1 complication was observed at a donor site $(8.3 \%)$. This was a minor incident involving a soft-tissue infection in which the primary closures were observed to have opened on the 5 th day after surgery. The wound was drained and resutured, and no further complications oc- 
curred. None of the patients experienced pain or difficulty in walking lasting longer than 2 weeks.

With regard to dental implant placement after the grafted sites had healed, no complications were observed. All dental implants that were placed at the grafted sites were osseointegrated well, and there was no implant failure during the course of the postoperative follow-up period.

\section{Discussion}

One of the most challenging tasks in oral and maxillofacial surgery is the reconstruction of the moderately to severely deficient maxillary or mandibular alveolar bone in preparation for placement of endosseous dental implants [2]. To date, autogenous bone remains the gold standard for alveolar reconstruction, as it possesses the quality of osteogenesis due to osteoprogenitor cells already present within the graft $[2,7,8]$.

When deciding on a donor site, defect size and shape are the most important parameters in determining the amount of bone graft to be harvested [9]. When larger alveolar augmentations and jaw reconstructions are undertaken, extraoral (distant) donor sites are preferred [2]. The iliac crest has traditionally been the preferred extraoral donor site in situations where preprosthetic or reconstructive needs call for bigger bone grafts [9]; however, complication and morbidity rates at this site are relatively high when compared to tibial bone-harvesting procedures [10]. The proximal tibia offers an excellent source of cancellous bone, and tibial bone harvesting for oral surgical reconstruction has been advocated by several authors $[2,3,5,6,10]$. Bone harvesting from the tibia for various reconstruction procedures in oral and maxillofacial surgery was originally introduced as an alternative to iliac crest harvesting [11]. In the literature, some studies have already compared complication rates and amounts of bone collected with tibial harvesting with those of iliac crest harvesting [11-13]. According to the literature, advantages to the tibia as a donor site include high amounts of cancellous bone availability with a less traumatic approach and with a low complication rate [11, 12]. Additionally, it is a technically quick and simple procedure, and also it allows for simultaneous harvesting and recipient site augmentation [2]. Moreover, unlike iliac crest graft harvesting which requires general anesthesia, bone harvesting from the tibia can be done as an outpatient procedure under local anesthesia even without sedation [11]. All in all, our results confirmed that bone har- vesting from the medial proximal tibia under a local anesthesia setting has been noted as a well-tolerated procedure by the patient. Our results showed that the tibial bone harvesting procedure is easy to conduct with lower complication rates and leading to predictable outcomes for various oral surgical reconstructions. These results are in agreement with the literature mentioned above.

The volume of bone available for harvesting from the proximal tibia is reported to be $25-40 \mathrm{~cm}^{3}$ of noncompressed cancellous bone [2]. However, Wang et al. [14] reported an average of $77 \mathrm{~cm}^{2}$ of cancellous bone available for harvesting in the proximal tibia, and their results showed this is $2-3$ times over the accepted average of bone volume. In this presented report, the authors easily managed to harvest $5.2 \pm 1.6 \mathrm{~cm}^{3}$ of tibial cancellous bone with a curette. According to the literature, a lot more than that amount of bone can easily be harvested from the proximal tibia without compromising the tibial metaphysis. However, we did not need to harvest more, and our results have confirmed the literature that moderate size reconstructions can predictably be performed using tibial bone as an outpatient procedure with low morbidity.

When iliac bone is compared to tibial bone as sources for bone grafts, there is a misperception that the quality of cancellous bone from the tibia is of inferior quality [2]. Clinically, in comparison to tibial bone, cancellous bone harvested from the iliac crest is known to be slightly denser [11]. This is because tibial bone has a higher amount of fat as compared to iliac crest bone [2]. This misperception has already been challenged by the study of Sivarajasingam et al. [15]. Their prospective study showed similar optical bone densities in tibial and iliac crest bone grafts over the first 3 postoperative months. With regard to results of the secondary alveolar cleft reconstruction of our study, the follow-up periods showed similar results with the other studies that showed good osseous healing of the cleft sites. None of the cases in our study $(n=4)$ had any complication regarding tibial bone grafting. Within the limitation of this study, we believe that the tibial bone can be a good alternative for the reconstruction of alveolar clefts compared to iliac bone.

Two different methods are used in harvesting bone grafts from the tibia, namely the medial and lateral approaches. While the classical approach to the cancellous bone in the proximal tibia is the lateral approach, the medial approach is considered alternate [2]. Herford et al. [5] conducted a study on cadavers comparing the lateral and the medial approaches, and they noted that both approaches yield almost equal amounts of cancellous 
graft. Although the mean volume of bone harvested from either approach is similar, the medial approach offers several advantages, such as easier access to the surgical site, less risk of damaging anatomical formations and less risk of postoperative morbidity $[2,5]$. The authors reported that the medial approach is advantageous as it is away from any vital anatomical structures, whereas the lateral approach is closer to vital structures such as the articular surface, the anterior tibialis muscle, and the recurrent tibial vessels, and is located within the anterior compartment [2]. Herford et al. [5] also reported that when utilizing the medial approach the bone is closer to the skin. For these reasons, the minimally invasive medial approach was used with all of the patients in the presented study. According to the presented study results, reaching the medial proximal tibia to harvest cancellous bone was easy and quick without encountering any vital anatomical structures. Our results are in agreement with the previous study reporting the bone is closer to the skin surface. When it comes to incision size, it relates to the size of the bony window on the proximal tibia and varies between the different studies ranging from 1 to $3 \mathrm{~cm}$ in length $[2,5,16]$. The incision with our technique for the medial approach is placed over a point $15 \mathrm{~mm}$ medial to the tibial parallel line and $15 \mathrm{~mm}$ superior to the tibial perpendicular line, and it is around $3 \mathrm{~cm}$ long. At this point, the thickness of the subcutaneous tissue is minimal, therefore this allows an almost through-andthrough incision with minimal bleeding and dissection. We noted that the accurate determination of the location of the tibial perpendicular line can be sometimes difficult since the determination of this line is based on the accurate finding of the tibial tuberosity, and finding of this location can be problematic in overweight individuals. In this study we used a 'trapdoor technique' along with a curette to harvest bone. To be able to utilize a curette within the proximal tibial metaphysis, we performed bony cuts of $1-2 \mathrm{~cm}$ by $1 \mathrm{~cm}$ for the 'trapdoor', and this comes with increased time and instrumentation when compared to the bone trephination technique. According to the literature when small quantities of bone are required, a bone trephination technique can be used with a smaller skin incision and potentially less morbidity [16]. According to our results, the medial approach to the proximal tibia is safe, quick and avoids any potential injury to the important structures. The medial approach does not require entry into any of the four lower extremity compartments; however, we suggest care should be taken when this approach is utilized by the novice surgeon.

Tibial Bone Grafting in Dental Surgery
According to the literature tibial bone harvesting is a relatively safe and simple procedure; however, it is not without potential complications [2]. The most frequently reported complications include gait disturbance, prolonged pain, infection, wound dehiscence, hypertrophic scarring, ecchymosis, hematoma, seroma, paresthesia, tibial fracture and violation of the joint space [2, 17-21]. According to this presented report, of the 12 consecutive cases only 1 patient $(8.3 \%)$ had a minor complication at the donor site. There were no major complications at all. At the recipient site, there were only 2 minor complications, and they were resolved with minor interventions. This report shows that the surgical approach was relatively simple and safe, the overall resorption rate of the graft was minimal, and the levels of postoperative pain and functional impairment (gait disturbance) were minimal. All the patients included in this study were ambulatory the same day without any problem. Our patients reported that they developed a temporary gait disturbance, which resolved within postoperative week 2 . In the literature one study reported a donor site complication rate of $1.3 \%$ in 230 proximal tibial harvests [20]. Another study reported a complication rate of $1.9 \%$ with 1 postoperative hematoma and no major complication [3]. Kushner [22] reported a complication rate of $1.4 \%$ with 1 wound dehiscence and 1 superficial infection in a series of 141 patients. With regard to complications, the results of this presented study showed good results similar to those of the current literature such as acceptable pain, swelling and gait disturbance following tibial harvest surgery. Additionally, there was no unacceptable scarring at the donor site. These results were also in agreement with the published studies.

Although the incidence of major complications such as proximal tibial fracture has been reported, there are few documented reports $[18,19]$. It has been suggested that the most major complications can be avoided by a careful operative technique and by being familiar with the pertinent anatomy. Additionally, the patient should avoid all movement in which a load is applied to the legs, especially sport activities, for at least 6 weeks following surgery, and the amount of bone harvested from the site should be kept within reasonable limits $[18,19]$. In the present study experienced surgeons did all the tibial harvest surgeries with a meticulous surgical technique. All patients were asked to avoid any strenuous physical activity to prevent tibial fracture for the postoperative 12 weeks. As a result we did not observe any major complications in our patient population, which undermines the reports saying major complications are rare for medial 
proximal tibial bone harvest and can be preventable with a good surgical technique.

In this study all transplanted tibial autogenous bone grafts were well integrated into the recipient sites (100\%). Our results showed that all dental implants placed at the reconstructed bony sites were osseointegrated well without any complications. When osseointegration and survival of dental implants are concerned, the best bone graft material is autogenous cancellous bone graft. Since our aim was to prepare deficient sites for dental implant placement, cancellous autogenous bone from the proximal tibia worked very efficiently and predictably for this purpose.

\section{Conclusion}

In this presented study, we showed that harvesting of the tibial bone for alveolar bone augmentation was a simple and safe procedure without major complications. The medial approach to the tibial bone seemed to offer the same advantages as the classic lateral approach with less morbidity compared to the lateral approach. Hence, we conclude that tibial bone harvesting can be safely performed in an outpatient or in-office setting, and therefore, we strongly suggest the use of tibial bone harvesting via the medial approach in the reconstruction of mild-tomoderate bone grafting procedures in the oral cavity.

\section{References}

1 Boyne PJ, Herford AS: An algorithm for reconstruction of alveolar defects prior to implant placement. Oral Maxillofac Surg Clin North Am 2001;13:533-538.

2 Zouhary KJ: Bone graft harvesting from distant sites: concepts and techniques. Oral Maxillofac Surg Clin North Am 2010;22:301-316.

- 3 Alt V, Nawab A, Seligson D: Bone grafting from proximal tibia. J Trauma 1999;47:555557.

4 Winsenburg B: The reconstruction of anterior residual bone defects in patients with cleft lip, alveolus and palate: a review. J Maxillofac Surg 1985;13:197-208.

5 Herford AS, King BJ, Audia F, et al: Medial approach for tibial bone graft: anatomic study and clinical technique. J Oral Maxillofac Surg 2003;61:358-363.

-6 Lezcano FJ, Cagigal BP, Cantera JM, et al: Technical note: medial approach for proximal tibia bone graft using a manual trephine. Oral Surg Oral Med Oral Pathol Oral Radiol Endod 2007;104:11-17.

7 Misch CE: Bone augmentation for implant placement: keys to bone grafting; in Misch CE (ed): Contemporary Implant Dentistry, ed 2. St Louis, Mosby, 1999, pp 51-467.

-8 Sàndor G, Rittemberg B, Clokie C, et al: Clinical success in harvesting autogenous bone using a minimally invasive trephine. J Oral Maxillofac Surg 2003;61:164-168.
-9 Gerressen M, Hermanns-Sachweh B, Riediger $\mathrm{D}$, et al: Purely cancellous vs corticocancellous bone in sinus floor augmentation with autogenous iliac crest: a prospective clinical trial. Clin Oral Implant Res 2009;20:109-115

10 Chen C-M, Chen P-L, Wu CW, et al: Proximal tibial bone harvesting under local anesthesia without intravenous sedation in the dental office: case report. Kaohsiung J Med Sci 2008;24:103-106.

11 Kirmeier R, Payer M, Lorenzoni M, et al: Harvesting of cancellous bone from the proximal tibia under local anesthesia: donor site morbidity and patient experience J Oral Maxillofac Surg 2007;65:2235-2241.

12 Catone GA, Reimer BL, McNeir D, et al: Tibia autogenous cancellous bone as an alternative donor site in maxillofacial surgery: a preliminary report. J Oral Maxillofac Surg 1992; 50:1258-1263.

13 Ilankovan V, Stronczek M, Telfer M, et al: A prospective study of trephined bone grafts of the tibial shaft and iliac crest. Br J Oral Maxillofac Surg 1998;36:434-439.

14 Wang K, Almeida LE, Olsson AB: Volume analysis of the proximal tibial metaphysis. J Oral Maxillofac Surg 2007;65:2425-2429.

15 Sivarajasingam V, Pell G, Morse M, et al: Secondary bone grafting of alveolar clefts; a densitometric comparison of iliac crest and tibial bone grafts. Cleft Palate Craniofac J 2001;38: $11-14$.
16 Hernández-Alfaro F, Martí C, Biosca MJ, et al: Minimally invasive tibial bone harvesting under intravenous sedation. J Oral Maxillofac Surg 2005;63:464-470.

17 Walker T, Modayil PC, Cascarini L, et al: Retrospective review of donor site complications after harvest of cancellous bone from the anteriomedial tibia. Br J Oral Maxillofac Surg 2009; 47:20-22.

18 Hughes CW, Revington PJ: The proximal tibia donor site in cleft alveolar bone grafting: experience of 75 consecutive cases. J Craniomaxillofac Surg 2002;30:12-17.

19 Thor A, Farzad P, Larsson S: Fracture of the tibia: complication of bone grafting to the anterior maxilla. Br J Oral Maxillofac Surg 2006; 44:46-48.

20 O'Keeffe RM, Riemer BL, Butterfield SL: Harvesting of autogenous cancellous bone graft from the proximal tibial metaphysis. A review of 230 cases. J Orthop Trauma 1991;5:469474 .

21 Lezcano FJ, Cagigal BP, Cantera JM, et al: Technical note: medial approach for proximal tibia bone graft using a manual trephine. Oral Surg Oral Med Oral Pathol Oral Radiol Endod 2007;104:e11-e17.

22 Kushner GM: Tibia bone graft harvest technique. Atlas Oral Maxillofac Surg Clin North Am 2005;13:119-126. 